\title{
Does Sporadic Amyotrophic Lateral Sclerosis Spread via Axonal Connectivities?
}

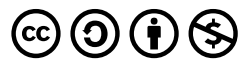

\author{
Authors
}

H. Braak' ${ }^{1}$, M. Neumann², 3, A. C. Ludolph ${ }^{4}$, K. Del Tredici ${ }^{1}$

\author{
Affiliations \\ 1 Clinical Neuroanatomy/Department of Neurology, Center \\ for Biomedical Research, University of Ulm \\ 2 Institute for Neuropathology, University Clinic Tübingen \\ 3 German Center for Neurodegenerative Diseases (DZNE), \\ Tübingen \\ 4 Department of Neurology, University of Ulm
}

Key words

$\alpha$-motor neuron, monosynaptic transmission, amyotrophic lateral sclerosis, Betz cells, TDP-43

\author{
Bibliography \\ ISSN 2511-1795 \\ Correspondence: \\ Prof. Heiko Braak, M.D. \\ Center for Biomedical Research \\ University of Ulm \\ Helmholtzstrasse 8/1 \\ $89081 \mathrm{Ulm}$ \\ Germany \\ heiko.braak@uni-ulm.de
}

DOI https://doi.org/10.1055/s-0043-111375

Neurology International Open 2017; 1: E136-E141

(c) Georg Thieme Verlag KG Stuttgart · New York

\begin{abstract}
The pathological process underlying sporadic amyotrophic lateral sclerosis (SALS) that is associated with the formation of cytoplasmic inclusions of a nuclear protein (TDP-43) is confined to only a few types of long-axoned projection neurons. The giant Betz pyramidal cells of the primary motor neocortex as well as large $\alpha$-motor neurons of the lower brainstem and spinal cord become involved early. In the human brain, these 2 neuronal types are to a large extent interconnected by monosynaptic axonal projections. The cell nuclei of affected neurons gradually forfeit their normal expression of the protein TDP-43. In $\alpha$-motor neurons, this nuclear loss is followed by the formation of insoluble TDP-43-immunopositive inclusions in the cytoplasm, whereas in Betz cells the loss of nuclear expression remains for an unknown period of time unaccompanied by somatodendritic and/or axoplasmic aggregations. It is possible that in cortical pyramidal cells (Betz cells) the nuclear clearing initially leads to the formation of an abnormal but still soluble cytoplasmic TDP-43 which may enter the axoplasm and, following transmission via direct synaptic contacts, induces anew TDP-43 dysregulation and aggregation in recipient neurons. The trajectory of the spreading pattern that consecutively develops during the course of SALS is consistent with the dissemination from chiefly cortical projection neurons via axonal transport through direct synaptic contacts leading to the secondary induction of TDP-43-containing inclusions within recipient nerve cells in involved subcortical regions.
\end{abstract}

\section{Introduction}

Amyotrophic lateral sclerosis (ALS) is characterized by rapid progressive paralysis of the striated skeletal musculature [1, 2]. The present review focuses on sporadic disease (sALS), which constitutes the majority of cases without a known genetic mutation. Clinically similar but inherited (familial) forms that display very diverse pathologies [3-7] are not taken into account here.

The pathological process underlying sALS entails abnormal changes of an endogenous and predominantly intranuclear protein, TDP-43 (transactive response DNA-binding protein 43). Following nuclear clearance, the protein remains delocalized in the cytoplasm of susceptible nerve cells, where it undergoes defective phosphorylation and conformational change followed by ubiquitination, which ultimately prevent its re-entry into the nuclear compartment [7-11].
The pathomechanisms that lead to the dysfunction and death of involved cells are still unknown. Along with a "loss of function" attributable to the loss of intranuclear TDP-43 expression accompanied by disrupted RNA metabolism, a "gain of function" mechanism owing to a noxious effect on cells by toxic TDP-43 forms has been discussed [7, 12]

\section{Which Nerve Cell Types Develop TDP-43-immunoreactive Inclusions in SALS?}

The abnormal nuclear clearance and development of cytoplasmic TDP-43-immunopositive inclusions are confined to a few types 
of nerve cells, and these have an axon that is, in comparison to the dimensions of the soma, disproportionately long. Projection neurons with a short axon do not become involved. Motor neurons are especially vulnerable. However, non-motor cells (e. g., projection neurons of the inferior olivary nucleus, the parvocellular portion of the red nucleus, and the striatum) are also susceptible $[13,14]$. Thus, the widely used term 'motor neuron disease' fails to encompass all aspects of the pathological process underlying SALS. The giant pyramidal cells of Betz in layer Vb of the primary motor neocortex and the large $\alpha$-motor neurons of the lower brainstem and of the spinal cord anterior horn become involved early and are especially hard hit ( $\triangleright$ Fig. 1) [14].

\section{Which Nerve Cell Types Are Protected against Such Inclusions?}

The resistance of many neuronal types to SALS is striking: Even among the motor neurons there are some that seldom or never develop TPD-43-positive lesions: for instance, the visceromotor preganglionic motor neurons of the dorsal glossopharyngeal and vagal nuclei as well as the parasympathetic and sympathetic nuclei in the spinal cord lateral horn [14]. Compared to the morphologically homogeneous group of somatomotor nerve cells in the spinal cord and in motor nuclei of the cranial nerves V, VII, and XI-XII that develop markedly severe pathology, the same cell type in the nuclei of the cranial nerves III, IV, and VI, which control the extrinsic eye muscles, remains virtually intact for the duration of the disease [14].

\section{Corticofugal Projections Control Suscepti- ble Neurons}

In humans, the somatomotor neurons of the lower brainstem and spinal cord are directly controlled by corticobulbar and corticospinal projections $[15,16]$, whereas the nuclei of the extrinsic eye muscles are only indirectly subject to influence by the cerebral cortex and receive their contacts essentially from noncortical fibers of the medial longitudinal fascicle $[17,18]$. Visceromotor neurons are subject to, above all, the control of noncortical autonomic centers and to the modulatory influence of the reticular formation and aminergic nuclei of the lower brainstem. As such, it is evident that the existence of direct (i. e., monosynaptic) control by the neocortex is one of the prerequisites for the affection of subcortical nerve cells in the sALs pathological process. In the same context, it should be pointed out that the group of so-called 'nonthalamic nuclei with diffuse projections' (i. e., the locus coeruleus, upper raphe nuclei, magnocellular nuclei of the basal forebrain) to the cerebral cortex

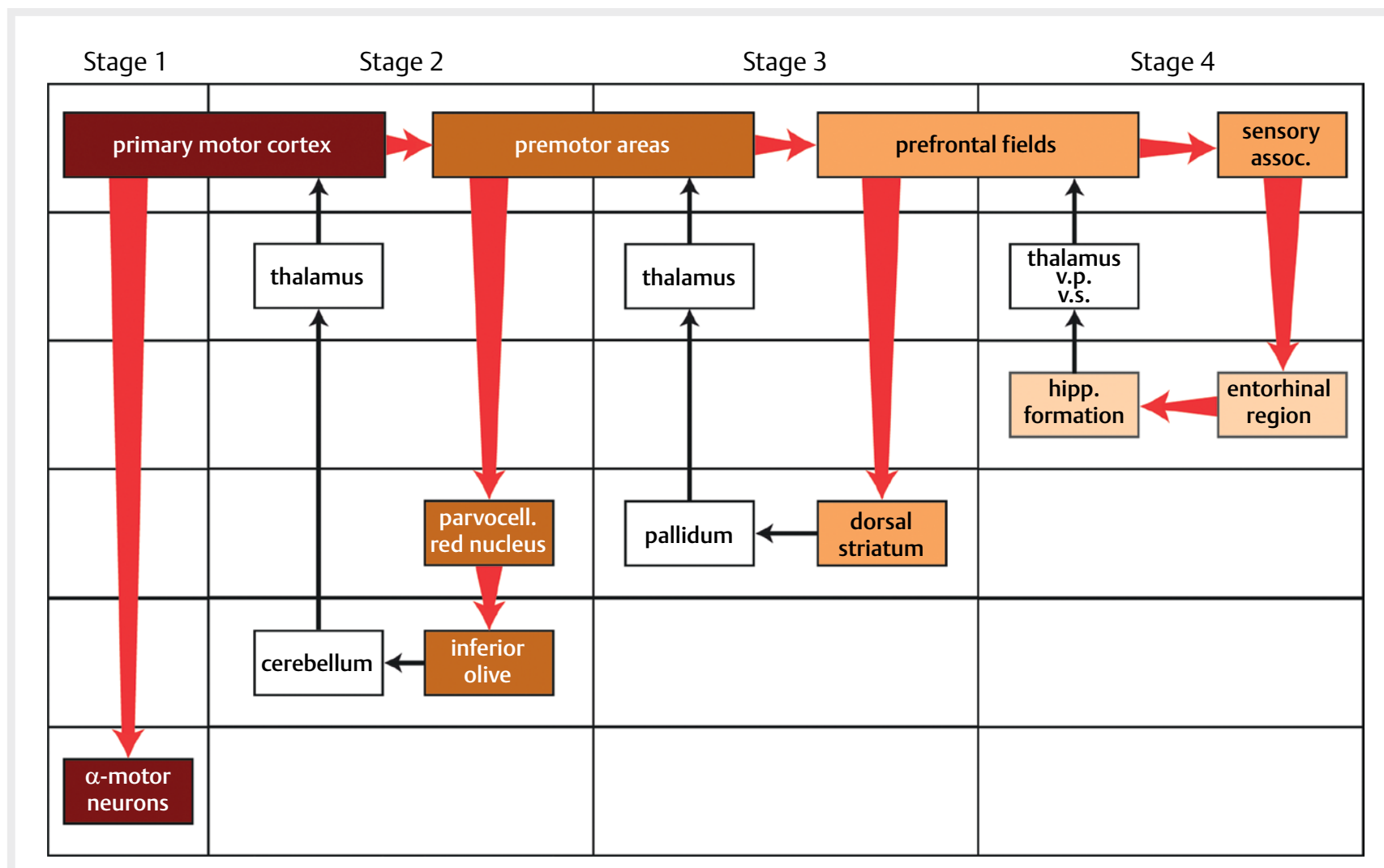

- Fig. 1 Simplified summary diagram of the regions in the human central nervous system that become involved during the course of sALS. The diagram indicates the progression of the pathological process in 4 stages within the neocortex (uppermost row) in relationship to the involvement of subcortical as well as allocortical areals which are interconnected with the neocortex via massive corticofugal projections (thick red arrows). Color coding: Stage 1: dark brown, Stage 2: light brown, Stage 3: dark orange, Stage 4: light orange. Abbreviations: hipp. form. - hippocampal formation; parvocell. red nucleus - red nucleus, parvocellular portion; sensor. assoc. - sensory association areas; v.p. - ventral pallidum; v.s. - ventral striatum. 
and to other regions that become severely involved in other neurodegenerative proteinopathies, such as Alzheimer's disease and Parkinson's disease, remain virtually intact in sALS cases. In other words, projection cells with predominantly corticopetal connectivities to the cerebral cortex, in contrast to those controlled by corticofugal projections, are not subject to pathological changes.

\section{The Transmission of the Pathology from Cortical Pyramidal Cells to Projection Neurons of Target Regions}

The inclusions that systematically develop during sALS display a characteristic topographical distribution pattern within the central nervous system. During the course of the disease, additional groups of neurons in previously uninvolved regions are drawn into the pathological process in a time-dependent manner. These changes in the distribution pattern of the lesions can be assigned to 4 neuropathological stages ( $\triangleright$ Fig. 1) $[14,19]$. Naturally, the duration and transi- tion of each stage cannot be determined within the context of post-mortem cross-sectional studies; nevertheless, in vivo diffusion-tensor imaging (DTI) and diffusion-weighted imaging (DWI)based results gained from ALS patients fit in well with the proposed hypothetical sequence of neuropathological staging [20-24].

Stage 1: Abnormal changes develop not only in Betz pyramidal cells in the deep layer $\mathrm{Vb}$ of the primary motor cortex (Brodmann field 4) [25] but also in lower brainstem and spinal cord $\alpha$-motor neurons ( $\triangleright$ Fig. 1,2) [3]. These changes commence during the first stage and continue during the subsequent stages.

Stage 2: In addition to those in the agranular motor cortex (chiefly Brodmann fields 4 and 6), lesions appear in adjacent portions of the prefrontal cerebral cortex. In the midbrain, the pathology develops in the parvocellular portion of the red nucleus, which is controlled directly via corticorubral projections [26] and, in the lower brainstem, in parts of the reticular formation and the precerebellar nuclei, such as the inferior olivary nucleus ( $\bullet$ Fig. 1). By contrast, the magnocellular portions of the red nucleus that project to the anterior horn of the spinal cord remain intact

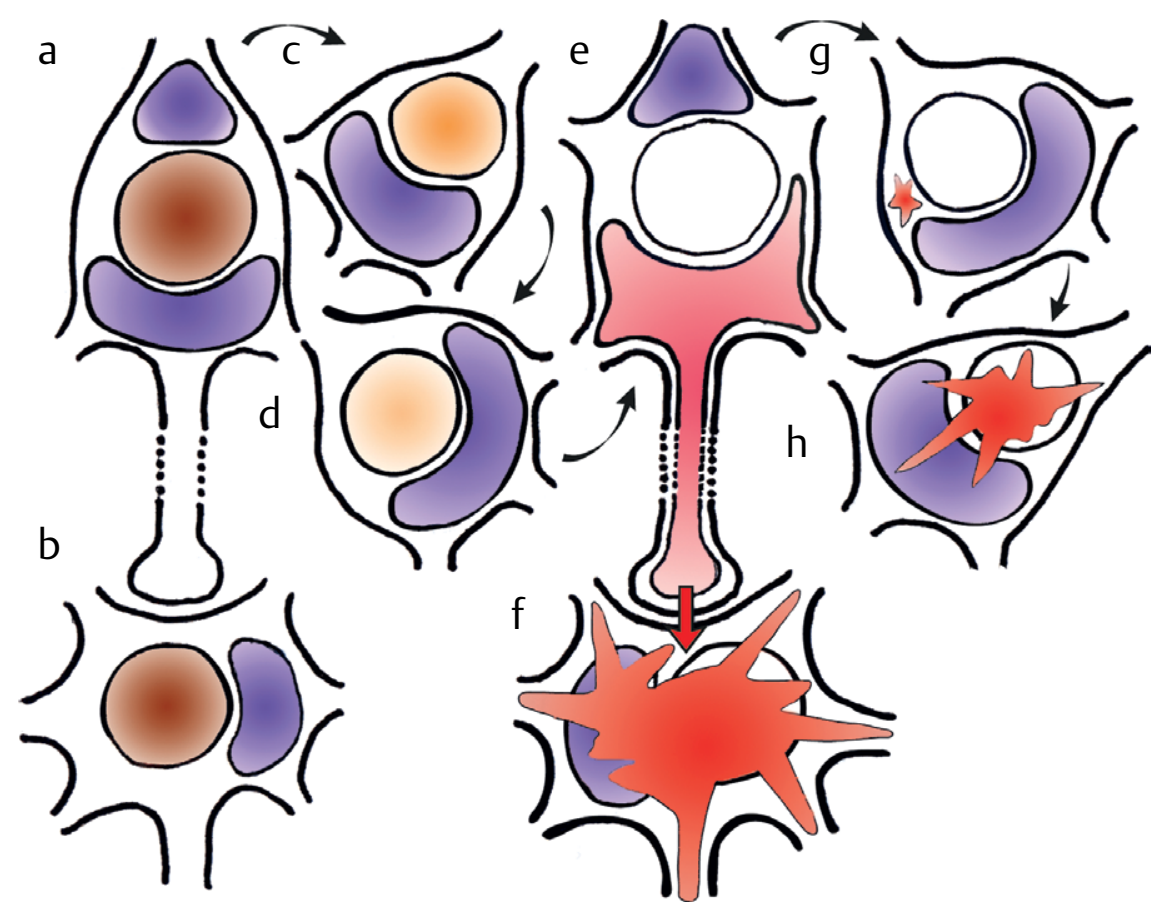

- Fig. 2 Summary diagram of the changes seen in Betz giant pyramidal cells (a, $\mathbf{c}-\mathbf{e}, \mathbf{g}, \mathbf{h}$ ) and in $\mathbf{\alpha}$-motor neurons (b, f) during sALS. The Betz cells of the human adult are characterized by large deposits of lipofuscin pigment granules that are represented here by violet shading. $\mathbf{a}$, $\mathbf{b}$. Betz cells in controls as well as noninvolved Betz cells and $\alpha$-motor neurons in SALS cases display strongly TDP-43 immunopositive intranuclear staining (here, in brown). The lengthy Betz cell axons project to and synapse directly (monosynaptically) on corresponding $\alpha$-motor neurons in the lower brainstem and or in the spinal cord ventral horn. c, d. Involved Betz cells display a gradual reduction in their TDP-43 intranuclear immunostaining. e. The result of this development is marked by a completely 'empty' (i. e., TDP-43-immunonegative) cell nucleus. In such instances, the abnormal protein probably is present for an indefinite time period in the somatodendritic and/or axonal cytoplasm in a soluble state (here, in pink). $\mathbf{g}, \mathbf{h}$. The presence of occasionally smaller or (seldom) larger TDP-43 aggregates may indicate that the Betz cells are fundamentally capable of producing insoluble inclusions out of the soluble (and presumably toxic) TDP-43 protein. $\mathbf{f}$. With the appearance of the Betz cell abnormalities (c-e), TDP-43 immunopositive aggregates also develop in the somatic and axonal cytoplasm of $\alpha$-motor neurons (see red blasts). These observations suggest that the spread of the pathological process may take place via axonal transport of soluble forms of abnormal TDP-43 and its subsequent monosynaptic transmission to corresponding $\alpha$-motor neurons. This transsynaptic spreading is indicated by means of the red arrow in the synapse between cells in $\mathbf{e}$ and $\mathbf{f}$. The dashed lines in $\mathbf{a}$ and $\mathbf{e}$ are intended to show that the Betz cell axons are much longer and thinner, in comparison to the size of their somata, than depicted here schematically. Reproduced with permission from Springer Press [32]. 
Stage 3. The pathology in the prefrontal fields becomes more extensive and reaches both the orbital gyri and gyrus rectus; via the superior longitudinal fascicles it also arrives at the postcentrally located sensory fields of the parietal and occipital lobes. At subcortical sites, the lesions develop in the medium-sized GABAergic projection neurons of the striatum ( $\vee$ Fig. 1 ). Within the 3 subnuclei there, the accumbens nucleus (ventral striatum) becomes involved first followed by the putamen and caudate nucleus [27]. The striatum receives strong corticostriatal projections originating in layer $\mathrm{V}$ of the neocortex, the putamen projections arising predominantly in areas of the agranular motor cortex, and the caudate nucleus projections coming from prefrontal fields.

Stage 4. The cortical pathology expands further and enters, via the neocortical regions of the temporal lobe, the transitional zone between the temporal neo- and allocortex (transentorhinal region) as well as adjacent portions of the allocortex (entorhinal region, hippocampal formation) ( Fig. 1) [14]. The allocortical regions of the temporal lobe that are responsible for learning and the consolidation of memory become involved relatively late [28].

It is remarkable that the hallmark pathological changes develop only in regions that receive and are under the direct control of strong corticofugal projections ( $\triangleright$ Fig. 1 ), whereas regions that chiefly project to the cerebral cortex, including the locus coeruleus and upper raphe group, are mostly spared [29]. This situation provides support for the idea that the driving force behind the origins and relentless progression of the TDP-43 pathology may reside in the cerebral cortex itself (beginning in the neocortical motor fields) mediated via corticofugal axons and the anterograde transport of soluble TDP-43 that is neurotoxic ( $\triangleright$ Fig. 1,2) [19, 30-32]. The damage inflicted on selected nerve cells of the cerebral cortex would be, for all intents and purposes, the source that necessarily leads to the subsequent pathological changes in regions of the lower brainstem and spinal cord innervated monosynaptically by these cortical neurons.

\section{The Pathology in Cortical Betz Cells Differs from That in Bulbar and Spinal $\alpha$-Motor Neurons}

The giant pyramidal cells of Betz and the large $\alpha$-motor neurons in spinal cord and lower brainstem have been well characterized morphologically. They are readily distinguishable from other cell types of the central nervous system and, thus, well-suited for delineating the development of the TDP-43 pathology in both neuronal types ( Fig. 2) [25].

Beginning in stage 1 , it is possible to see alongside of the many normal Betz cells and $\alpha$-motor neurons displaying strong TDP-43-positive intranuclear staining an increasing number of the same cell types with reduced intranuclear TDP-43 immunostaining. In time and with disease progression, the nuclei of such cells completely lack TDP-43 immunoreactivity. However, many of the involved Betz cells also fail to display any TDP-43-positive cytoplasmic inclusions ( $\triangleright$ Fig. 2) [32]. Only closer scrutiny of a larger number of these cells reveals the presence of a few that contain very subtle cytoplasmic aggregates. Thus, it can be concluded that cortical Betz cells do not completely forfeit their capacity to produce insoluble cyptoplasmic inclusions; on the contrary, this capacity is only suppressed for an indeterminate interval of time or the process of TDP-43 aggregation occurs at a remarkably slow rate ( $\mathbf{F i g} . \mathbf{2}$ ).

The nuclei of the large bulbar and spinal cord $\alpha$-motor neurons also display the phenomena of reduced TDP-43-positive intranuclear staining and, ultimately, complete TDP-43 intranuclear clearance. In contrast to the Betz cell population, however, the $\alpha$-motor neurons with weak TDP-43-immunoreactive nuclei contain needle-like cytoplasmic aggregates that extend into the somatodendritic and axonal compartments of involved cells [33]. Then, the aggregates appear to coalesce into coarser skein-like inclusions ( Fig. 2) [32].

\section{Can the Pathology Be Propagated Transsynaptically from the Cortical Projection Neurons to Interconnected Projection Neurons?}

In the ascending primate scale, the direct control of corticofugal tracts via monosynaptic contacts increasingly replaces the indirect influence exerted on the $\alpha$-motor neurons by the cerebral cortex via intervening interneurons in lower mammals. In the human nervous system, this development reaches a provisional acme $[15,16,29,32,34]$. The assumption that cortical neurons (Betz cells) are the first to become involved followed by the $\alpha$-motor neurons, tends to favor Charcot's theory [35], namely, that ALS originates in the lateral funiculus (i. e., corticospinal tract, where the axons of the Betz cells are located) and only then affects the $\alpha$-motor neurons in the anterior horn of the spinal cord [36]. The sources of the very first malfunctions within the protein TDP-43 in Betz cells are incompletely understood; but, it is conceivable that the further spread of the pathology to interconnected neurons takes place by means of the phylogenetically 'new' or recent monosynaptic contacts [9, 29, 32, 37-41]. Whether the same holds true not only for corticobulbar and corticospinal projections but also for corticorubral and corticostriatal connectivities is currently an open question.

\section{Conclusions}

We have postulated for SALS that the misfolded and abnormally phosphorylated protein TDP-43 may be present in the cytoplasm in a soluble phase in involved Betz cells displaying nuclear clearance (TDP-43 immunonegative cell nuclei) and a TDP-43-immunonegative cytoplasm. Anterograde axonal transport of the soluble protein could permit it to reach the presynaptic terminals of the Betz cells, where its transsynaptic (cell-to-cell) transmission to the corresponding $\alpha$-motor neurons of the spinal cord and brainstem could lead anew therein to the pathological dysregulation and prion-like seeded propagation of TDP-43 [42]. According to this hypothesis, the emergence of cytoplasmic aggregates within target neurons would mostly interfere with the ensuing spread of the pathology to the immediately following neuron in the neuronal chain ( Fig. 1).

The regional distribution pattern of the TDP-43 pathology described above is not compatible with theories of a pathological process that purportedly commences in motor neurons of the spinal 
cord or lower brainstem, followed, secondarily, by damage to cortical areas [43-47]: Such a proposed route of progression fails to explain the involvement in SALS of the parvocellular portion of the red nucleus and of the medium-sized GABAergic projection neurons in the striatum, none of which have any connectivities whatsoever to spinal or bulbar motor neurons ( $\mathbf{F i g . ~ 1 ) . ~}$

The concept that the disease process can be primarily influenced by the cerebral cortex is corroborated by the clinical findings reported for split-hand syndrome and split-leg phenomenon [4854]. Clinical sALS phenotypes that eventually allow for alternative interpretations, e. g., flail-arm or flail-leg syndrome [45-47, 55], still require neuropathological confirmation $[56,57]$.

From this standpoint, it is essential to elucidate mechanisms that might preserve TDP-43 for an indeterminate period of time in an abnormal but soluble phase within the cytoplasm of cortical projection cells and to clarify the mechanisms by means of which neuron-to-neuron transmission occurs. Selective intervention into these mechanisms could eventually prevent the TDP-43 pathology from spreading into interconnected motor neurons.

\section{Acknowledgement}

The authors wish to thank Mr. David Ewert (University of Ulm) for skillful technical assistance with the graphics.

\section{Conflict of interest}

No conflict of interest has been declared by the authors.

\section{References}

[1] Kiernan MC, Vucic S, Cheah BC et al. Amyotrophic lateral sclerosis. Lancet 2011; 377: 942-955

[2] Ludolph A, Drory V, Hardiman O et al. A revision of the El Escorial criteria - 2015. Amyotroph lateral scler frontotemporal degener 2015; 16: 291-292

[3] Al-Chalabi A, Jones A, Troakes C et al. The genetics and neuropathology of amyotrophic lateral sclerosis. Acta Neuropathol 2012; 124: 339-352

[4] Rosen DR, Siddique T, Patterson D et al. Mutations in Cu/Zn superoxide dismutase gene are associated with familial amyotrophic lateral sclerosis. Nature 1993; 362: 59-62

[5] Kwiatkowski T] Jr, Bosco DA, Leclerc AL et al. Mutations in the FUS/TLS gene on chromosome 16 cause familial amyotrophic lateral sclerosis. Science 2009; 323: 1205-1208

[6] Vance C, Rogelj B, Hortobagyi T et al. Mutations in FUS, an RNA processing protein,cause familial amyotrophic lateral sclerosis type 6 . Science 2009; 323: 1208-1211

[7] Ratti A, Buratti E. Physiological functions and pathobiology of TDP-43 and FUS/TLS proteins. J Neurochem 2016; 138 (Suppl 1): 95-111

[8] Ayala YM, Zago P, D’Ambrogio D et al. Structural determinants of the cellular localization and shuttling of TDP-43. J Cell Sci 2008; 121: 3778-3785

[9] Lee S, Kim H]. Prion-like mechanism in amyotrophic lateral sclerosis: Are protein aggregates the key? Exp Neurobiol 2015; 24: 1-7
[10] Neumann M, Sampathu DM, Kwong LK et al. Ubiquitinated TDP-43 in frontotemporal lobar degeneration and amyotrophic lateral sclerosis. Science 2006; 314: 130-133

[11] Neumann M, Kwong LK, Lee EB et al. Phosphorylation of $\$ 409 / 410$ of TDP-43 is a consistent feature in all sporadic and familial forms of TDP-43 proteinopathies. Acta Neuropathol 2009; 117: 137-149

[12] Lee EB, Lee VMY, Trojanowski JQ. Gains or losses: Molecular mechanisms of TDP-43-mediated neurodegeneration. Nat Rev Neurosci 2011; $13: 38-50$

[13] Piao YS, Wakabayashi K, Kakita A et al. Neuropathology with clinical correlations of sporadic amyotrophic lateral sclerosis: 102 autopsy cases examined between 1962 and 2000. Brain Pathol 2003; 13: $10-22$

[14] Brettschneider ], Del Tredici K, Toledo JB et al. Stages of pTDP-43 pathology in amyotrophic lateral sclerosis. Ann Neurol 2013; 74: 20-38

[15] Lemon RN. Descending pathways in motor control. Ann Rev Neurosci 2008; 31: 195-218

[16] Lemon RN. What drives corticospinal output? F1000 Biol Rep 2010; 2: 51

[17] Büttner-Ennever JA. The extraocular motor nuclei: Organization and functional neuroanatomy. Progr Brain Res 2006; 151: 95-125

[18] Horn AK, Leigh RJ. The anatomy and physiology of the ocular motor system. Hand Clin Neurol 2011; 102: 21-69

[19] Fatima M, Tan R, Halliday GM et al. Spread of pathology in amyotrophic lateral sclerosis: Assessment of phosphorylated TDP-43 along axonal pathways. Acta Neuropathol Commun 2015; 3: 47

[20] Kassubek J, Müller H-P, Del Tredici K et al. Diffusion tensor imaging analysis of sequential spreading of disease in amyotrophic lateral sclerosis confirms patterns of TDP-43 pathology. Brain 2014; 137: $1733-1740$

[21] Müller HP, Turner MR, Grosskreutz J et al. A large-scale multicentre cerebral diffusion tensor imaging study in amyotrophic lateral sclerosis. J Neurol Neurosurg Psychiatry 2016; 87: 570-579

[22] Schulthess I, Gorges M, Müller H-P et al. Functional connectivity changes resemble patterns of pTDP-43 pathology in amyotrophic lateral sclerosis. Sci Rep 2016; 6: 38391

[23] Schmidt R, de Reus MA, Scholtens LH et al. Simulating disease propagation across white matter connectome reveals anatomical substrate for neuropathology staging in amyotrophic lateral sclerosis. Neurolmage 2016; 124: 762-769

[24] Hornberger M, Kiernan MC. Emergence of an imaging biomarker for amyotrophic lateral sclerosis: Is the end point near? J Neurol Neurosurg Psychiatry 2016; 87: 569

[25] Kushchayev SV, Moskalenko VF, Wiener PC et al. The discovery of the pyramidal neurons: Vladimir Betz and a new era of neuroscience. Brain 2012; 135: 285-300

[26] Habas C, Cabanis EA. Cortical projections to the human red nucleus: A diffusion tensor tractography study with a 1.5-T MRI machine. Neuroradiol 2006; 48: 755-762

[27] Zhang H, Tan CF, Mori F et al. TDP-43 immunoreactive neuronal and glial inclusions in the neostriatum in amyotrophic lateral sclerosis with and without dementia. Acta Neuropathol 2008; 115: 115-122

[28] Wightman G, Anderson VER, Martin J et al. Hippocampal and neocortical ubiquitin-immunoreactive inclusions in amyotrophic lateral sclerosis with dementia. Neurosci Lett 1992; 139: 269-274

[29] Braak H, Brettschneider ], Ludolph AC et al. Amyotrophic lateral sclerosis - a model of corticofugal axonal spread. Nat Rev Neurol 2013; 9: 708-714

[30] Fallini C, Bassell G, Rossoll W. The ALS disease protein TDP-43 is actively transported in motor neuron axons and regulates axon outgrowth. Hum Mol Genet 2012; 21: 3708-3718 
[31] Saper CB, Wainer BH, German DC. Axonal and transneuronal transport in the transmission of neurological disease: Potential role of system degenerations, including Alzheimer's disease. Neuroscience 1989; 23 : 389-398

[32] Braak H, Ludolph AC, Neumann M et al. Pathological TDP-43 changes in Betz cells differ from those in bulbar and spinal $\alpha$-motoneurons in sporadic amyotrophic lateral sclerosis. Acta Neuropathol 2017; 133: 79-90

[33] Braak H, Ludolph AC, Thal DR et al. Amyotrophic lateral sclerosis: Dash-like inclusions of phosphorylated TDP-43 in somatodendritic and axonal compartments of somatomotor neurons of the lower brainstem and spinal cord. Acta Neuropathol 2010; 120: 67-74

[34] Eisen A, Weber M. The motor cortex and amyotrophic lateral sclerosis. Muscle Nerve 2001; 24: 564-579

[35] Charcot J-M. Sclérose latéral amyotrophique. In: œuvres complètes. Vol 2: Paris: Bureaux du Progrès Médical; 1874: 249-266

[36] Jin J, Hu F, Zhang $Q$ et al. Hyperintensity of corticospinal tract tract on FLAIR: A simple and sensitive objective upper motor neuron degeneration marker in clinically verified amyotrophic lateral sclerosis. J Neurol Sci 2016; 367: 177-183

[37] Feiler MS, Strobel B, Freischmidt A et al. TDP-43 is intercellularly transmitted across axon terminals. J Cell Biol 2015; 211: 897-911

[38] Grad LI, Fernando SM, Cashman NR. From molecule to molecule and cell to cell: Prion-like mechanisms in amyotrophic lateral sclerosis. Neurobiol Dis 2015; 77: 257-265

[39] Guo L, Shorter J. Biology and pathobiology of TDP-43 and emergent therapeutic strategies. Cold Spring Harb Perspect Med 2016 pii:a024554

[40] Smethurst P, Sidle KCL, Hardy J. Review: Prion-like mechanisms of transactive response DNA binding protein of $43 \mathrm{kDa}$ (TDP-43) in amyotrophic lateral sclerosis (ALS). Neuropathol Appl Neurobiol 2015; 41: 578-597

[41] Smethurst P, Newcombe ], Troakes $C$ et al. In vitro prion-like behaviour of TDP-43 in ALS. Neurobiol Dis 2016; 96: 236-247

[42] Kanouchi T, Ohkubo T, Yokota T. Can regional spreading of amyotrophic lateral sclerosis motor symptoms be explained by prion-like propagation? J Neurol Neurosurg Psychiatry 2012; 83: 739-745

[43] Ravits JM, La Spada AR. ALS motor phenotype heterogeneity, focality, and spread: Deconstructing motor neuron degeneration. Neurology 2009; 73: 805-811
[44] Talman P, Duong T, Vucic S et al. Identification and outcomes of clinical phenotypes in amyotrophic lateral sclerosis/motor neuron disease: Australian National Motor Neuron Disease observational cohort. BJM Open 2016; 6: e012054

[45] Gamez J, Cervera C, Codina A. Flail arm syndrome of Vulpian-Bernhart's form of amyotrophic lateral sclerosis. J Neurol Neurosurg Psychiatry 1999; 67: 258

[46] Wijesekera C, Mathers S, Talman P et al. Natural history and clinical features of the flail arm and flail leg ALS variants. Neurology 2009; 72 : 1087-1094

[47] Schuster C, Kasper E, Machts J et al. Focal thinning of the motor cortex mirrors clinical features of amyotrophic lateral sclerosis and their phenotypes: A neuroimaging study. J Neurol 2013; 260: 2856-2864

[48] Wilbourn A]. The "split hand syndrome”. Muscle Nerve 2000; 23: 138

[49] Simon NG, Lee M, Bae JS et al. Dissociated lower limb muscle involvement in amyotrophic lateral sclerosis. J Neurol 2015; 262: 1424-1432

[50] Weber M, Eisen A, Stewart $\mathrm{H}$ et al. The split hand in ALS has a cortical basis. J Neurol Sci 2000; 180: 66-70

[51] Menon P, Bae JS, Mioshi E et al. Split-hand plus sign in ALS: Differential involvement of the flexor pollicis longus and intrinsic hand muscles. Amyotroph Lateral Scler Frontotemporal Degener 2013; 14: 315-318

[52] Menon P, Kiernan MC, Vucic S. Cortical dysfunction underlies the development of the split-hand in amyotrophic lateral sclerosis. PLoS One 2014; 9: e87124

[53] Menon P, Kiernan MC, Vucic S. ALS pathophysiology: Insights from the split- hand phenomenon. Clin Neurophysiol 2014; 125: 186-193

[54] Eisen A, Turner MR, Lemon R. Tools and talk: An evolutionary perspective on the functional deficits associated with amyotrophic lateral sclerosis. Muscle Nerve 2014; 49: 469-477

[55] Menon P, Geevasinga N, Yiannikas C et al. Cortical contributions to the flail leg syndrome: Pathophysiological insights. Amyotroph Lateral Scler Frontotemporal Degener 2016; 17: 389-396

[56] Sasaki S, Iwata M. Motor neuron disease with predominantly upper extremity involvement: A clinicopathological study. Acta Neuropathol 1999; 98: 645-650

[57] Hino S, Sasaki S. Flail arm syndrome with cytoplasmic vacuoles in remaining anterior horn motor neurons: A peculiar variant of amyotrophic lateral sclerosis. Neuropathology 2015; 35: 582-586 